\title{
Benchmarking the NEWUOA on the BBOB-2009 Noisy Testbed
}

\author{
Raymond Ros \\ Univ. Paris-Sud, LRI \\ UMR 8623 / INRIA Saclay, projet TAO \\ F-91405 Orsay, France \\ raymond.ros@Iri.fr
}

\begin{abstract}
The NEWUOA which belongs to the class of DerivativeFree optimization algorithms is benchmarked on the BBOB2009 noisy testbed. A multistart strategy is applied with a maximum number of function evaluations of $10^{4}$ times the search space dimension.
\end{abstract}

\section{Categories and Subject Descriptors}

G.1.6 [Numerical Analysis]: Optimization-global optimization, unconstrained optimization; F.2.1 [Analysis of Algorithms and Problem Complexity]: Numerical Algorithms and Problems

\section{General Terms}

Algorithms

\section{Keywords}

Benchmarking, Black-box optimization, Derivative-free optimization

\section{ALGORITHM PRESENTATION}

The NEWUOA (New Unconstrained Optimization Algorithm) [4] is a Derivative-Free Optimization (DFO) algorithm using the trust region paradigm. NEWUOA computes a quadratic interpolation of the objective function in the current trust region and performs a truncated conjugate gradient minimization of the surrogate model in the trust region. It then updates either the current best point or the radius of the trust region. based on the a posteriori interpolation error. The time complexity of the algorithm is $\mathcal{O}\left(m^{2} n\right)$ in the worst case but in practice closer to $\mathcal{O}(m n)$, where $m$ is the number of interpolation points used for the determination of the quadratic model and $n$ is the dimension of the search space. The number of interpolation points $m$ is a parameter of the algorithm and needs to be chosen in the range $\left[n+2, \frac{(n+1)(n+2)}{2}\right]$. Other parameters of the algorithm

Permission to make digital or hard copies of all or part of this work for personal or classroom use is granted without fee provided that copies are not made or distributed for profit or commercial advantage and that copies bear this notice and the full citation on the first page. To copy otherwise, to republish, to post on servers or to redistribute to lists, requires prior specific permission and/or a fee.

GECCO'09, July 8-12, 2009, Montréal Québec, Canada

Copyright 2009 ACM 978-1-60558-505-5/09/07 ...\$5.00 are the initial and final radii of the trust region, respectively governing the initial 'granularity' and the precision of the search. A simple stochastic independent restart procedure (as advised in [2]) was added to improve the probability of the algorithm reaching a given target function value.

\section{EXPERIMENTAL PROCEDURE}

The implementation used for our experiments is the one provided by Matthieu Guibert ${ }^{1}$ which delivers Powell's original Fortran source code of the algorithm. This Fortran code has been integrated with the BBOB experimental paradigm. In this paper, the maximum number of points $m=\frac{(n+1)(n+2)}{2}$ has been used. Though the scaling of the algorithm time complexity is close to $\mathcal{O}\left(n^{4}\right)$, preliminary experiments showed the full model to deal with noisy functions better than a smaller model. The initial radius $\rho_{\text {beg }}$ of the search region has been set to 10 , the range of the search space. Preliminary experiments shows very few dependencies on this parameter, given it is not too small (ie. by many orders of magnitude) for the problem considered. A final radius $\rho_{\text {end }}=10^{-16}$ was chosen close to the limit being the machine precision to prevent numerical errors. The starting point $x_{0}$ is chosen uniformly in $[-5,5]^{n}$ where $n$ denotes the dimension. The multistart strategy was used with at most 100 restarts to reduce the duration of an experiment. For the same reason, a run is limited to at most $10^{4} \times n$ function evaluations. The algorithm used is presented in Figure 1. No parameter tuning was done, the CrE [2] is computed to zero.

\section{RESULTS AND DISCUSSION}

Results from experiments according to [2] on the benchmarks functions given in $[1,3]$ are presented in Figures 2 and 3 and in Tables 1 and 2. The algorithm solves some of the moderate noise function $f_{101}, f_{102}, f_{103}, f_{104}, f_{106}$. Else, $f_{105}, f_{107}, f_{109}, f_{112}, f_{113}, f_{115}, f_{125}, f_{127}, f_{128}, f_{130}$ are solved only for dimensions 2 or 3 . Noise greatly affects such trust region method, especially the uniform noise model.

\section{CPU TIMING EXPERIMENT}

For the timing experiment, the proposed algorithm was run on $f_{8}$ and restarted until at least 30 seconds have passed (according to Figure 2 in [2]). The experiments were conducted with an Intel Core 26700 processor $(2.66 \mathrm{GHz})$ on Linux 2.6.24.7. The results were 200, 86, 45, 7.9, 3.7, 36

\footnotetext{
${ }^{1}$ http://www.inrialpes.fr/bipop/people/guilbert/ newuoa/newuoa.html
} 


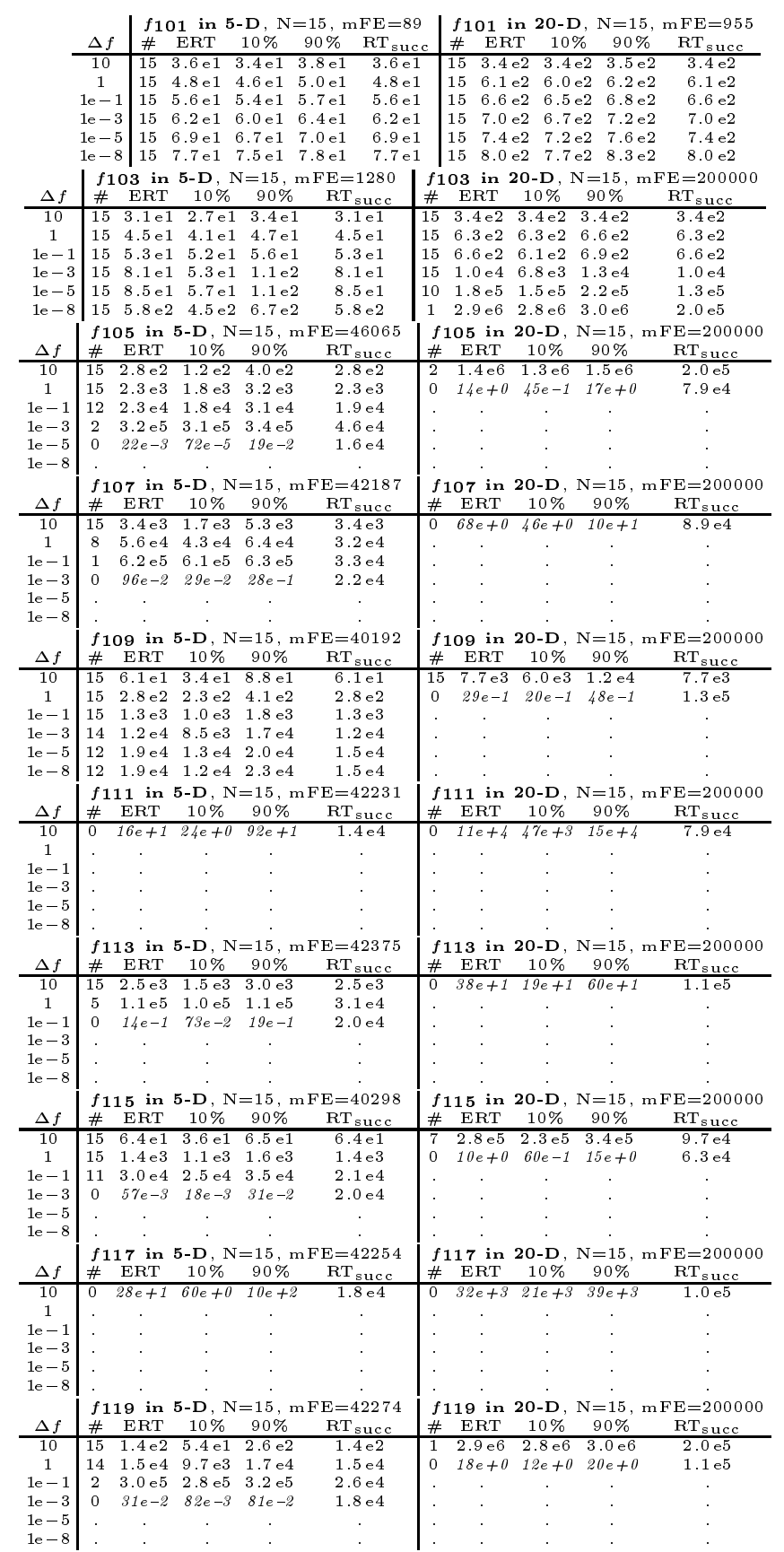

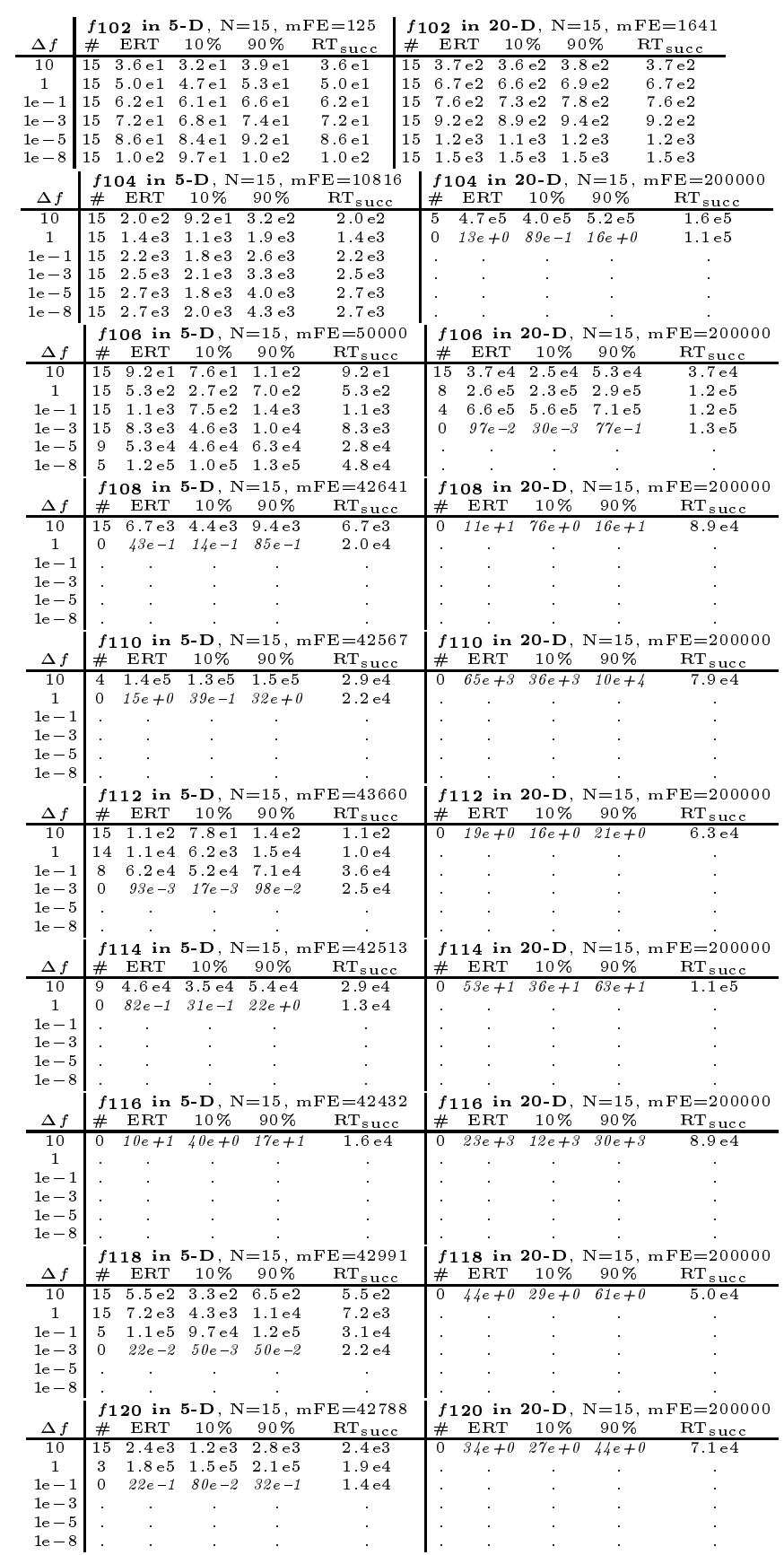

Table 1: Shown are, for functions $f_{101}-f_{120}$ and for a given target difference to the optimal function value $\Delta f$ : the number of successful trials (\#); the expected running time to surpass $f_{\mathrm{opt}}+\Delta f$ (ERT, see Figure 2); the $10 \%$-tile and $90 \%$-tile of the bootstrap distribution of ERT; the average number of function evaluations in successful trials or, if none was successful, as last entry the median number of function evaluations to reach the best function value $\left(\mathbf{R T}_{\text {succ }}\right)$. If $f_{\mathrm{opt}}+\Delta f$ was never reached, figures in italics denote the best achieved $\Delta f$-value of the median trial and the $10 \%$ and $90 \%$-tile trial. Furthermore, $\mathbf{N}$ denotes the number of trials, and mFE denotes the maximum of number of function evaluations executed in one trial. See Figure 2 for the names of functions. 


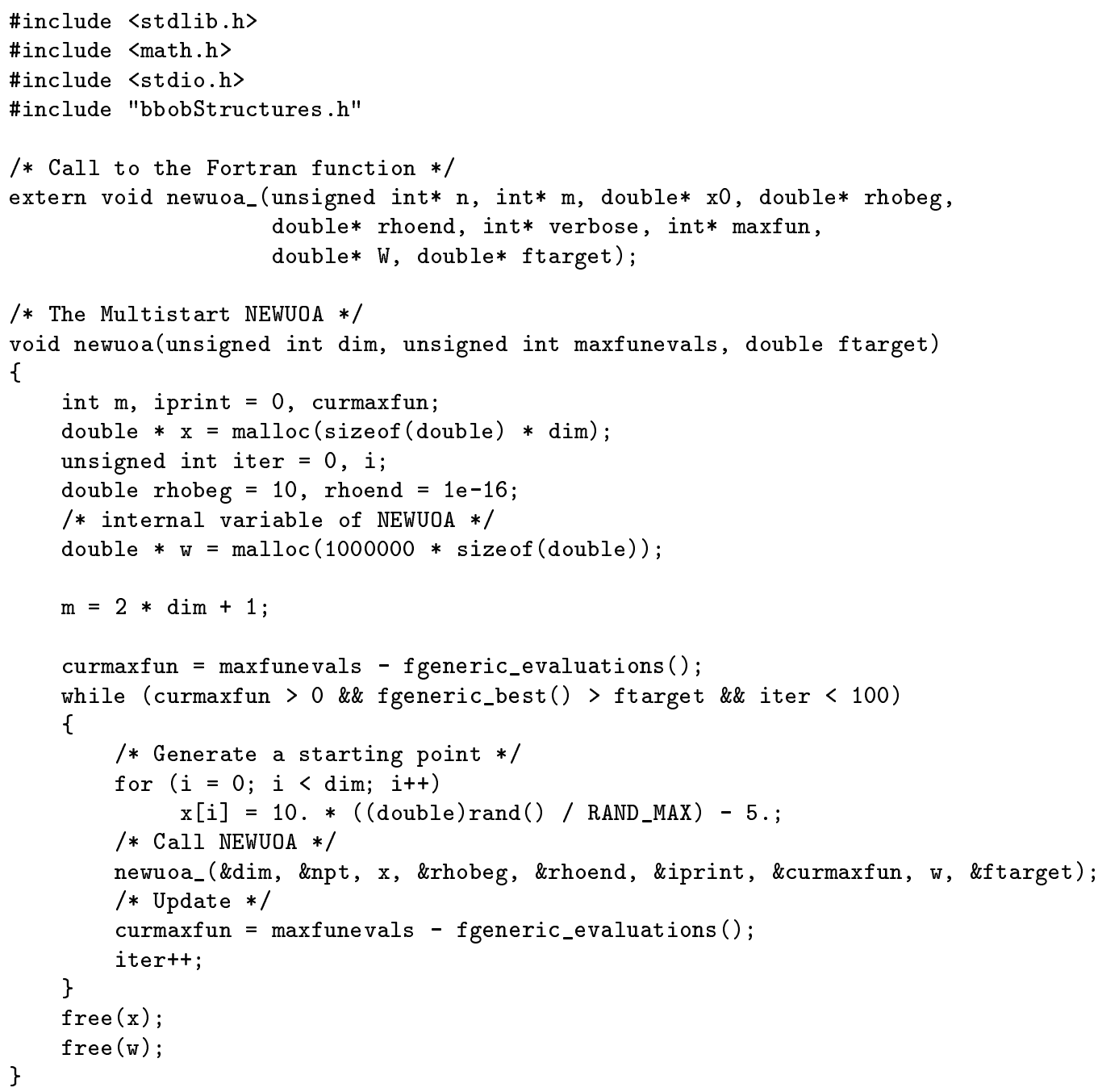

$\times 10^{-3}$ seconds per function for the full model evaluations in dimension $2,3,5,10,20$ and 40 respectively.

\section{CONCLUSION}

The NEWUOA which is a trust region method was tested with restarts on a noisy testbed. Method based on interpolation are expected to fail on noisy functions. Results of this algorithm do not disagree with this.

\section{Acknowledgments}

The first author would like to acknowledge the support, help, and work of the BBOB team with particular kudos to Anne Auger, Steffen Finck and Nikolaus Hansen.

\section{REFERENCES}

[1] S. Finck, N. Hansen, R. Ros, and A. Auger. Real-parameter black-box optimization benchmarking 2009: Presentation of the noisy functions. Technical Report 2009/21, Research Center PPE, 2009.
[2] N. Hansen, A. Auger, S. Finck, and R. Ros. Real-parameter black-box optimization benchmarking 2009: Experimental setup. Technical Report RR-6828, INRIA, 2009.

[3] N. Hansen, S. Finck, R. Ros, and A. Auger. Real-parameter black-box optimization benchmarking 2009: Noisy functions definitions. Technical Report RR-6869, INRIA, 2009.

[4] M. J. D. Powell. The NEWUOA software for unconstrained optimization without derivatives. Large Scale Nonlinear Optimization, pages 255-297, 2006. 

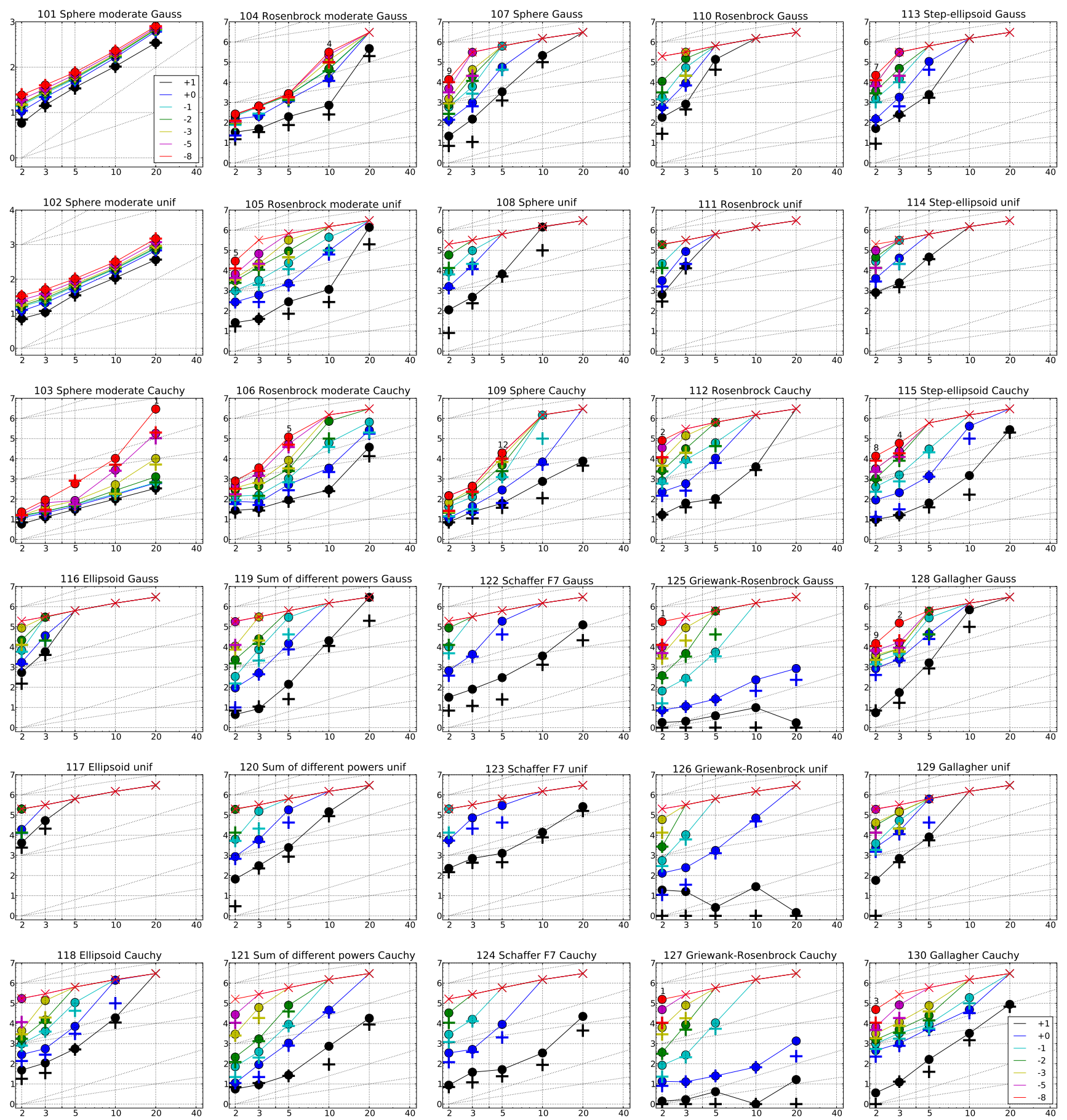

Figure 2: Expected Running Time (ERT, •) to reach $f_{\mathrm{opt}}+\Delta f$ and median number of function evaluations of successful trials $(+)$, shown for $\Delta f=10,1,10^{-1}, 10^{-2}, 10^{-3}, 10^{-5}, 10^{-8}$ (the exponent is given in the legend of $f_{101}$ and $\left.f_{130}\right)$ versus dimension in $\log$-log presentation. The $\operatorname{ERT}(\Delta f)$ equals to \#FEs $(\Delta f)$ divided by the number of successful trials, where a trial is successful if $f_{\mathrm{opt}}+\Delta f$ was surpassed during the trial. The \#FEs $(\Delta f)$ are the total number of function evaluations while $f_{\mathrm{opt}}+\Delta f$ was not surpassed during the trial from all respective trials (successful and unsuccessful), and $f_{\mathrm{opt}}$ denotes the optimal function value. Crosses $(\times)$ indicate the total number of function evaluations \#FEs $(-\infty)$. Numbers above ERT-symbols indicate the number of successful trials. Annotated numbers on the ordinate are decimal logarithms. Additional grid lines show linear and quadratic scaling. 

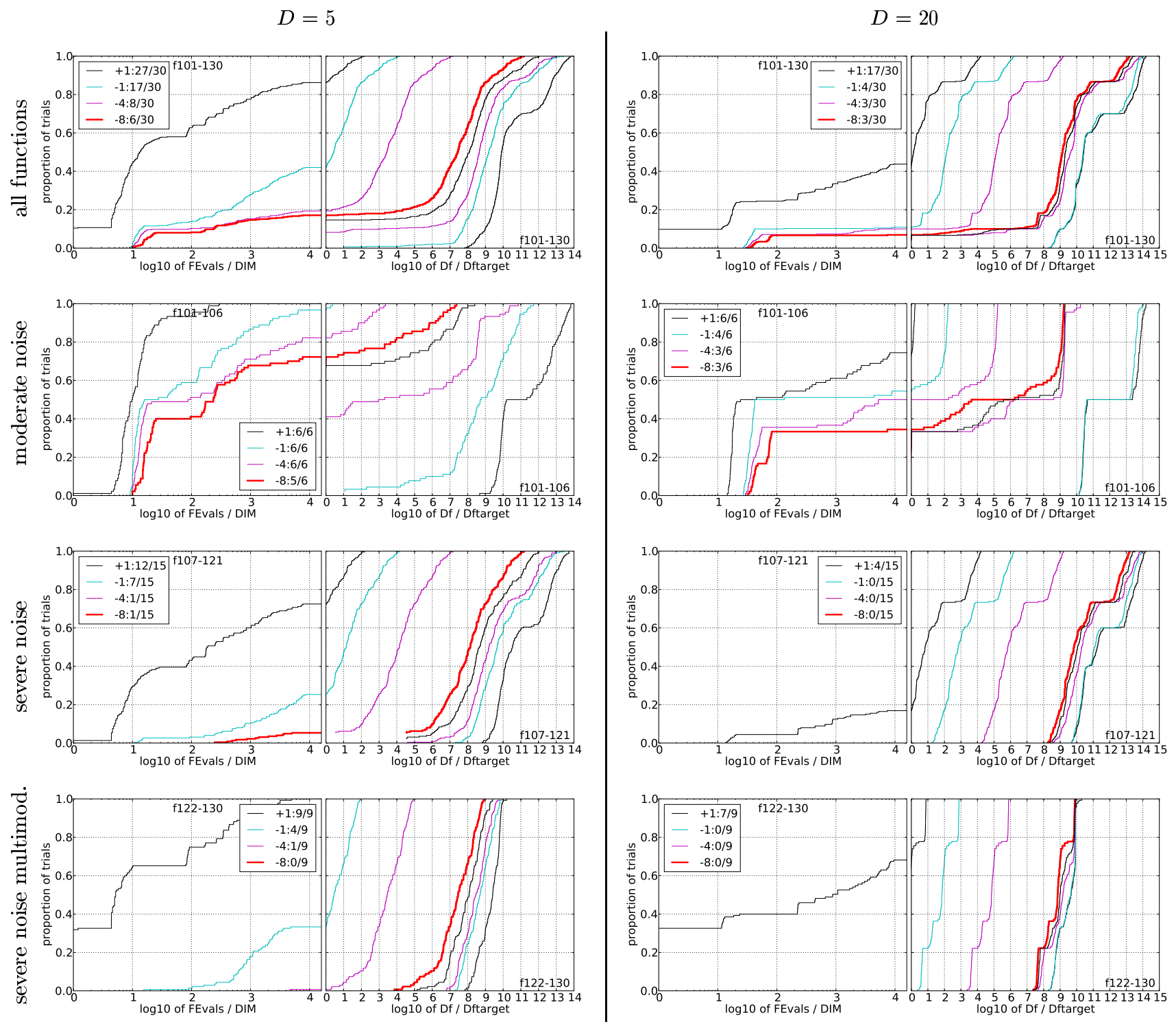

Figure 3: Empirical cumulative distribution functions (ECDFs), plotting the fraction of trials versus running time (left subplots) or versus $\Delta f$ (right subplots). The thick red line represents the best achieved results. Left subplots: ECDF of the running time (number of function evaluations), divided by search space dimension $D$, to fall below $f_{\mathrm{opt}}+\Delta f$ with $\Delta f=10^{k}$, where $k$ is the first value in the legend. Right subplots: ECDF of the best achieved $\Delta f$ divided by $10^{k}$ (upper left lines in continuation of the left subplot), and best achieved $\Delta f$ divided by $10^{-8}$ for running times of $D, 10 D, 100 \mathrm{D} \ldots$ function evaluations (from right to left cycling blackcyan-magenta). Top row: all results from all functions; second row: moderate noise functions; third row: severe noise functions; fourth row: severe noise and highly-multimodal functions. The legends indicate the number of functions that were solved in at least one trial. FEvals denotes number of function evaluations, $D$ and DIM denote search space dimension, and $\Delta f$ and Df denote the difference to the optimal function value. 

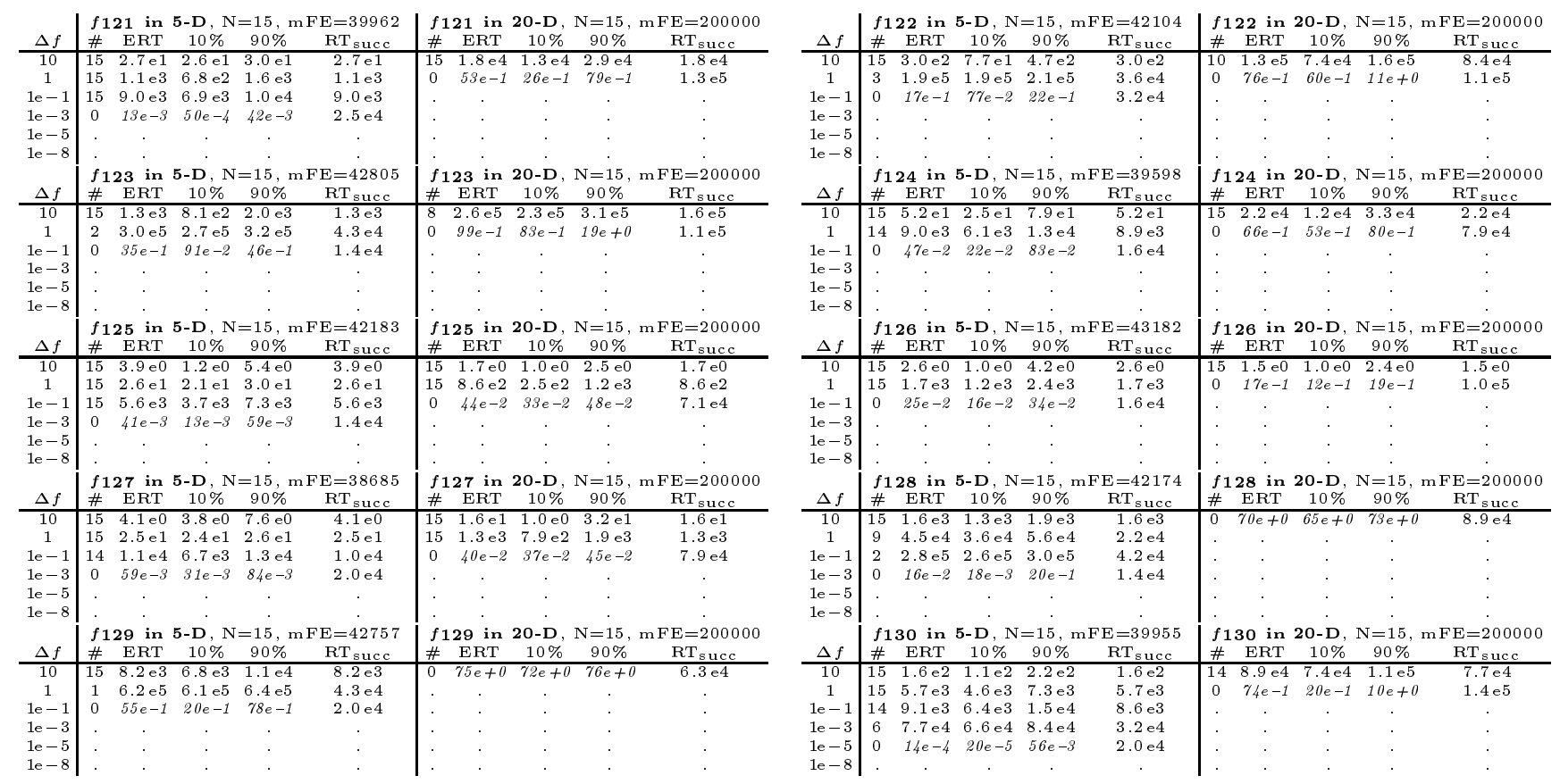

Table 2: Shown are, for functions $f_{121}-f_{130}$ and for a given target difference to the optimal function value $\Delta f$ : the number of successful trials (\#); the expected running time to surpass $f_{\mathrm{opt}}+\Delta f$ (ERT, see Figure 2); the 10\%-tile and $90 \%$-tile of the bootstrap distribution of ERT; the average number of function evaluations in successful trials or, if none was successful, as last entry the median number of function evaluations to reach the best function value $\left(\mathbf{R T}_{\text {succ }}\right)$. If $f_{\mathrm{opt}}+\Delta f$ was never reached, figures in italics denote the best achieved $\Delta f$-value of the median trial and the $10 \%$ and $90 \%$-tile trial. Furthermore, $\mathrm{N}$ denotes the number of trials, and mFE denotes the maximum of number of function evaluations executed in one trial. See Figure 2 for the names of functions. 